\title{
Isolation of Photosystem I Reaction Center Subunit K Gene GjPsaK From Gardenia jasminoides
}

\author{
Lan Gao ${ }^{1}$, Hao-Ming $\mathrm{Li}^{2}$ \\ School of Basic Courses \\ Guangdong Pharmaceutical University, Guangzhou Higher Education Mega Center \\ Guangzhou 510006, Peoples Republic of China \\ 1'glgdpu@aliyun .com, ${ }^{2}$ Corresponding author, Lihaoming@gdpu.edu.cn
}

\begin{abstract}
Objective: Photosystem I (PS I ) is a large membrane supercomplex that consists of a reaction centre and light-harvesting complex (LHC I ). PS I catalyzes lightdriven electron transport to produce reducing power NADPH, and then NADPH are used in the dark reactions of photosynthesis to fix $\mathrm{CO} 2$ and synthesize organic molecules. In this work, we will isolate gene of a reaction centre subunit Psak in Gardenia jasminoides. Methods: A Gardenia jasminoides fruit cDNA library was constructed, and the GjPsak cDNA was isolated from the cDNA library by sequencing method. Results: The cDNA was 593 bp in length, contains a predicted 393 bp ORF that encodes 130 amino acids, the gene was designated GjPsak. The deduced amino acid sequence showed homology to PsaK from plants. A three-dimensional model of GjPsaK was built; the structure is similar to structures of Plant PsaK. GjPsaK contains two transmembrane $\alpha$-helices connected by a positively charged stromal loop; both the $\mathrm{N}$ and $\mathrm{C}$ termini are located in the luminal side of thylakoid membrane. Conclusion: According to functions of PsaK in plant, we implicated that the GjPsaK would involved in state transition and stabilization of LHC I organization.
\end{abstract}

Keywords-photosynthesis component; photosystem I reaction center subunit $K$; cloning; Gardenia jasminoides; cDNA library.

\section{INTRODUCTION}

PS I is a multiprotein complex within the thylakoids, it uses light energy to transport electrons from the lumenal soluble electron carrier plastocyanin across the membrane to the stromal soluble electron acceptor ferredoxin. The reduced ferredoxin is subsequently used for NADPH production. Photosystems I have a core complex (reaction center) and an outer antenna moiety. The core complex, responsible for charge separation and the first steps of electron transport; and the peripheral antenna system LHC I (light-harvesting proteins antenna complex I ), which plays a role in light harvesting and the transfer of excitation energy to the reaction center. The core complex centers are widely conserved among organisms, and antenna systems are more variable.

The PS I in plants is composed of the core complex plus 4 Lhca's ( four subunits of LHC I ), the core complex of plant PSI is mostly composed of 12 subunits, PsaA-L;
The chlorophyll a and carotene are mainly associated with the two largest subunits PsaA and PsaB. Several improved crystallographic structural model of plant photosystem I was reported [1-4], these model was based on PS I - $\mathrm{LHCl}$ super-complex of Pea (Pisum sativum, garden pea) at 2.8

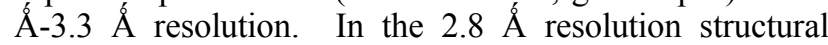
model, the positions of 12 core protein subunits (PsaA-L) bound with four light-harvesting proteins Lhca1-4, 156 chlorophylls, 32 carotenoids, 14 lipids, and 3 Fe4S4 clusters are identified, providing the most detailed picture of a plant PSI. PsaK is located near PsaA side of the core complex, contains two transmembrane $\alpha$-helices.

PsaK is a peripheral chlorophyll binding subunit; the position within the complex is at the junction where LHC I is expected to bind. PsaK was shown to regulate the disconnection of the LHC I antenna from PS I in Chlamydomonas reinhardtii cells subjected to irondeficiency conditions [5]. Light harvesting complex II (LHC II ) involved the regulation of distribution of light energy between PS II and PS I, which after phosphorylation can move from PS II to PS I in the socalled state 1 to state 2 transitions, and can move back to PS II after dephosphorylation. Part of the LHC II moves to PS I to from the PS I -LHC I - LHC II supercomplex and the supercomplex was purified rescently [6, 7], quenching of excess energy by PSI might be one of the physiological functions of PSI-PSII complexes [8]. The LHC II trimer is associated with the core on the opposite side of the Lhcas via the PsaH subunit. PsaK might have a role in state transitions, in Arabidopsis, the PS I -LHC II complex at $16 \AA$ resolution electron microscopy image showed that LHC II has a specific binding site at the PS I $-\mathrm{A} / \mathrm{H} / \mathrm{L}$, and PS I $-\mathrm{K}$ subunits [9]. The model of interaction between PS I and LHC II fits quite well with the possible interaction between the PsaK loop and the $\mathrm{N}$ terminus of LHCII [1]. It was reported that downregulation of the PsaK gene leads to higher Plum pox virus (PPV) accumulation in Nicotiana benthamiana, the cylindrical inclusion (CI) was found to interact with the PS I -K subunits, suggesting its role for PPV infection [10].

Gardenia jasminoides originates in Asia and has been in cultivation for at least a thousand years. The fruit of G. jasminoides is used in Asian countries as a natural colorant, and as a traditional herbal medicine. The orange or red colors of the fruit are caused by apocarotenoids, mainly 
crocin and crocetin, which are also two of active ingredients of this herbal medicine $[11,12]$. The PS I of $G$. jasminoides has not been elucidated, here we identified the PS I core subunit PsaK, and its structure was analyzed.

\section{MATERIALS AND METHODS}

\section{A. Plant and Growth Conditions}

G.jasminoides plants cultivated at Guangdong Pharmaceutical University were used as materials. Fruits were collected at development stage II , closed with yellowish green exocarp and orange mesocarp. The samples were stored at $-80^{\circ} \mathrm{C}$ until required.

\section{B. CDNA Library Construction, ESTs Sequencing and Cloning of GLhcb}

Total RNA was extracted from Gardenia fruit (stage II ), using a modified CTAB (hexadecyl trimethyl ammonium bromide) based extraction protocol [13]. From total RNA, the cDNA library construction and amplification were performed following the users manual of the CreatorTM SMARTTM cDNA Library construction Kit (Clontech, USA). The SMART cDNAs were ligated into SfiI-digested pDNR-LIB vector and transformed into Escherichia coli strain DH5 $\alpha$. Colonies were randomly picked, inoculate each colony to a separated PCR reaction solutions. The colony was lysised by heating the mixed
ESTs. There are 40 ESTs were sequenced. After sequencing and analysis, the colony containing the predicted pDNR-LIB-GjPsaK was isolated.

\section{RESULTS AND DISCUSSION}

To identify gene encoding subunit $\mathrm{K}$ of photosystem I in Gardenia jasminoides, a cDNA library of fruit was constructed and screened with primers M13 (see method). The inserts of about 200 clones were sequenced and analyzed. The full-length GjPsaK cDNA was then obtained (Genbank accession No. GU797552). The cDNA was $593 \mathrm{bp}$ in length, contains a predicted 393bp ORF that encodes 130 amino acids, a 72 bp non-coding region at $5^{\prime}$ end and a 128 bp of non-coding region flank at $3^{\prime}$ end. The predicted amino acid sequence of GjPsaK specifies a polypeptide with a molecular mass of $13.2 \mathrm{kDa}$ and a $\mathrm{pI}$ of 11.06 .

Protein sequence aligment indicates that the deduced GjPsaK protein is quite similar to other known PsaKs in plants; and the sequence homology is relatively lower with algae and cyanobacteria (Fig.1). GjPsaK had an $85.38 \%$ identity with PsaK in Vitis vinifera, $78.46 \%$ identity with Arabidopsis thaliana, 64.96\% identity with Oryza sativa, $61.43 \%$ identity with Picea wilsonii, $64.23 \%$ identity with Hordeum vulgare, $42.86 \%$ identity with Chlamydomonas reinhardtii and $18.46 \%$ identity with Cyanobacterium aponinum. The PsaK of Hordeum vulgare have two

\begin{tabular}{|c|c|}
\hline ja & 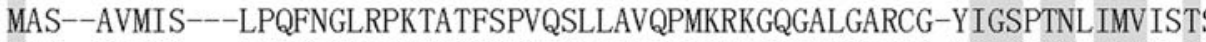 \\
\hline ( & MAA--TMMTS---LPQFNGLKPKTFSTSPVQGLVAVQPMRRKGQGALGARCD-FIGSPTNLI \\
\hline & MAS--TMMTT---LPQFNGLRATKISAAPVQGLASVQPMRRKGNGALGAKCD-FIC \\
\hline & MASQLSAMTS---VPQFHGLRS----YSSPRSMATLPSLRKR---SQGIRCD- \\
\hline & MASQLSAATS---VPQFHGLRT----YSSPRSMVTLPSLRMSKKRSQGIRCD-Y \\
\hline & MAS--TMMTT---LPQFNGLKPQPFSASPIQGLVAIQPRRNKGQGGLGARCD-FIGSSTNLI \\
\hline & MAA--TTMMTATALPQFSGLRP---SAAPVKSLAAVQPTRRKGNGALGARMD-FIGSPTNLIM \\
\hline & MQALAT--------------RP-----------SAIRPTKAARRSSVVVRADGFIGSSTNLIM \\
\hline & HGLRT-----YSSPRSMATLPSLRRRR--SQGIRCD-YIGSSTN \\
\hline & KLEI \\
\hline & -- \\
\hline & -DSGLQTGDPAGFTLA \\
\hline & SANRKATAGLKLEAR--ESGLQTGDPAGFTLADTLACGSFGHILGVGIVLGLKNTGVLDQIIG \\
\hline & SANRKSTAGLKLEAR--DSGLQTGDPAGFTLADTLACGAVGHIMGVGVVLGLKNIGVLDQIIG \\
\hline & SANRKATAGLKLEVR--DSGLQTGDPAGFTLADTLACGTVGHIIGVGVVLGLKNIGAL------ \\
\hline & IGFTLADTLACGSVGHIIGVGVVLGLKNIGAL----- \\
\hline & AGHGLGVGIVLGLKGIGAL----- \\
\hline & $\begin{array}{l}\text { TLADTLACGAVGHIMGVGIVLGLKNTGVLDQIIG } \\
\text { HНHНHНHHHHHHHHHНHH }\end{array}$ \\
\hline
\end{tabular}

Figure 1.Alignment of GjPsak with 8 protein sequences from plants and green algae. The abbreviation and Genbank accession numbers are: Gja (Gardenia jasminoides, ADE42969), Nta (Nicotiana tabacum, AAP03873), Ath (Arabidopsis thaliana, NP 174327), Tur (Triticum urartu, EMS60576), Osa (Oryza sativa, NP_001058895), Sly (Solanum lycopersicum, XP_004244537), Ptr (Populus trichocarpa, XP_002309626), Cre (Chlamydomonas reinhardtii, XP_001697230) and Hvu (Hordeum vulgare, PSAK_HORVU). Underlined, chloroplast transit peptide in Hvu; Alpha helices of the Hvu PsaK are marked with H. Identical residues are shaded.

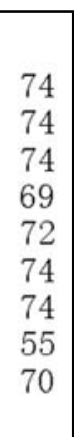

solutions at $95^{\circ} \mathrm{C}$ in a PTC-200 Thermocycler (MJ Research, USA) for $5 \mathrm{~min}$. After then, went to PCR amplification procedure with M13 primers provided by the CreatorTM SMARTTM cDNA Library construction Kit. The amplified PCR products (ESTs, expressed sequence tags) were analyzed by $1.2 \%$ agarose gel electrophoresis. When the amplified PCR products were longer than 1000 $\mathrm{bp}$, incubated the isolated colonies and sequenced the transmembrane $\alpha$-helices, while other sequences in Fig.1 show homology with the PsaK of Hordeum vulgare, and have similar loop region between the two $\alpha$-helices; with more divergence in their $\mathrm{N}$-terminal regions, there is a chloroplast transit peptide of 42 amino acids in Hordeum vulgare. 


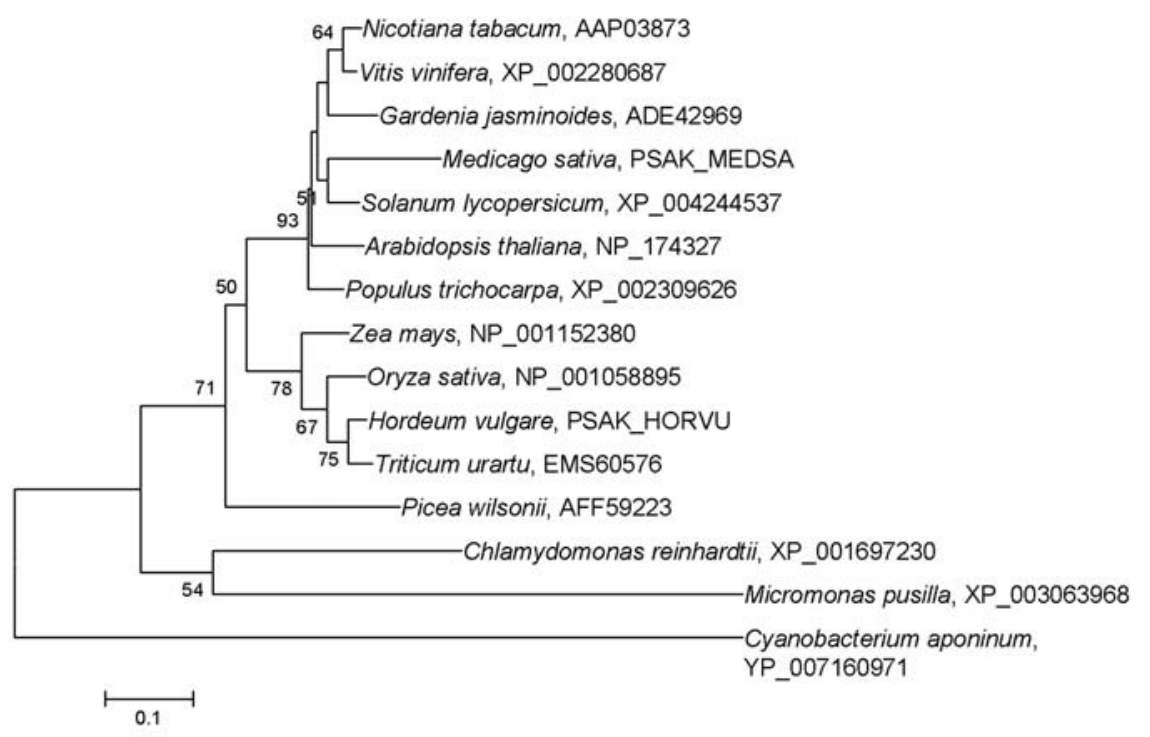

Figure 2 Phylogeny of 15 PsaK proteins. The Genbank accession numbers are indicated. The bootstrap values are shown in the tree nodes ( $\mathrm{n}=1000$, when consistency was poor, are not shown).

A phylogenetic tree was constructed for 15 sequences (Fig.2). Sequences were aligned using clustalW2. Phylogenetic trees were generated using MEGA4. The tree was built using neighbor-joining algorithm approach. From the tree in figure 2, it's showed that GjPsaK is closer to PsaK from plants than algae and cyanobacteria.

On the basis of the $3.3 \AA$ crystal structure of Pea PS I (PDB code 2WSF). The three dimension model structure of GjPsaK (Genbank accession no. ADE42969) was predicted using SWISS-PDB software [1]. The amino acid sequence of $\mathrm{GjPsaK}$ (from 47-130 residue range) has $83.33 \%$ amino acid identity with $2 \mathrm{WSF}$ (from 43-126 residue range). The structure was successfully built as a single chain. GjPsaK has the characteristic PsaK fold, both the $\mathrm{N}$ and $\mathrm{C}$ termini are located in the luminal side of thylakoid membrane, and between the two transmembrane $\alpha$-helices is a positively charged stromal loop (Fig. 3). The $3 \mathrm{D}$ structure of GjPsaK is similar to $2 \mathrm{WSF}$.

In the PS I supercomplex 4 Lhca's are associated with the core forming half a ring on the side of PsaF/J. PsaK located on the PsaA side of the core complex, near the PsaD and PsaL; and close to Lhca 4. Lhca 1 protein is bound to the core through PsaB and PsaG. Lhca 4 and Lhca 2 through small contact surfaces of PsaF and PsaJ interact with PS I core, respectively. Lhca 3 binds to PsaA and PsaK, and PsaK also have amino acid contacts with PsaA [4]. PsaK was proposed to stabilize LHC I organization. In Chlamydomonas reinhardtii, in adaptation to Fe-deficiency, the LHC I antenna uncoupled from PS I core followed by specific degradation of LHC I, it is proposed that disconnection is mediated by a change in the
A

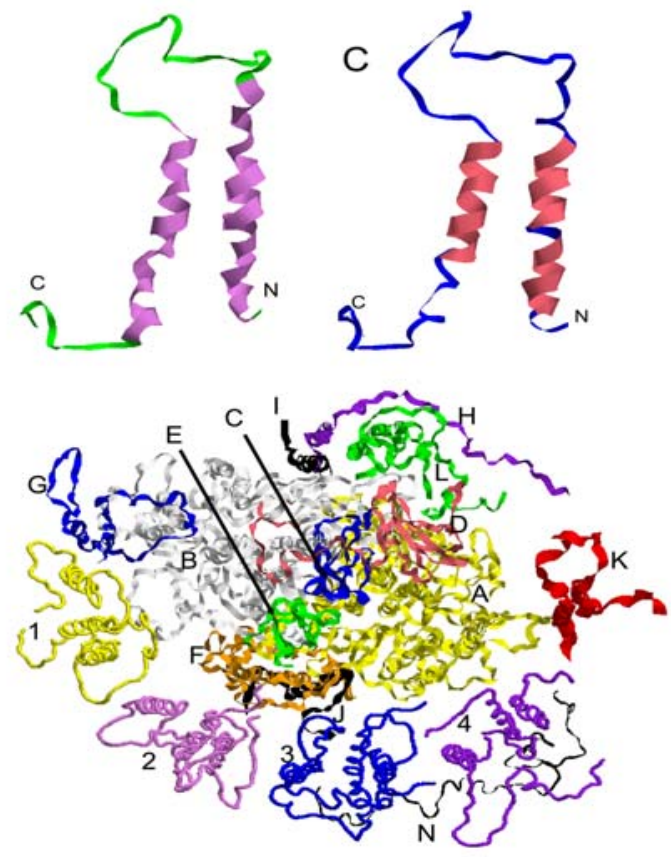

Figure 3 Structure of PsaK and the position of PsaK in plant PS I . (A) PsaK in PS I Crystal structure (from 43-126 residue range; PBD code 2wsf), (B) Plant PS I, view from the stroma. 1-4: Lhca's, are shown as trace diagram; A-L and N: core subunits, are shown as ribbon diagram. (C) Structure model of GjPsaK (from 47-130 residue range) In (A) and (C): View along the membrane. Conserved transmembrane $\alpha$-helixes are shown as violet and pink ribbon diagram respectively.

physical properties of PsaK [6]. In Arabidopsis, if lacking PsaK, there was $30-40 \%$ less Lhca 3 in PS I -LHC I complex [14]. 


\section{ACKNOWLEDGMENT}

This work was partially financially supported by the Guangdong Sci. \& Tech. Program (2013B010404046).

\section{REFERENCES}

[1] A. Amunts, H. Toporik, A. Borovikova and N. Nelson, "Structure determination and improved model of plant photosystem I," J Biol. Chem, Vol. 285, Jan. 2010, pp. 3478-3486, doi: 10.1074/jbc.M109.072645.

[2] A. Amunts, O. Drory and N. Nelson, "The structure of a plant photosystem I supercomplex at 3.4 A resolution ," Nature, Vol. 447, May. 2007, pp. 58-63.

[3] R. Croce, "Structural biology. A close view of photosystem I," Science, Vol. 348, May. 2015, pp. 970-971, doi: $10.1126 /$ science.aab3387.

[4] Y. Mazor, A. Borovikova and N. Nelson, "The structure of plant photosystem I super-complex at $2.8 \AA \AA$ resolution,” Elife, Jun. 2015, in press. doi: 10.7554/eLife. 07433 .

[5] J. L. Moseley, T. Allinger, S. Herzog, P. Hoerth, E. Wehinger, S. Merchant, et al., "Adaptation to Fe-deficiency requires remodeling of the photosynthetic apparatus,” EMBO. J, Vol. 21, Dec. 2002, pp. 6709-6720.

[6] P. Galka, S. Santabarbara, T. T. Khuong, H. Degand, P. Morsomme, R. C. Jennings, et al., "Functional analyses of the plant photosystem I-light-harvesting complex II supercomplex reveal that light-harvesting complex II loosely bound to photosystem II is a very efficient antenna for photosystem I in state II," Plant Cell, Vol. 24, Jul. 2012, pp. 2963-2978, doi: 10.1105/tpc.112.100339.
[7] E. Wientjes, H. van Amerongen and R. Croce, "LHCII is an antenna of both photosystems after long-term acclimation," Biochem. Biophys. Acta, Vol. 1827, Mar. 2013, pp. 420-426, doi: 10.1016/j.bbabio.2012.12.009.

[8] M. Yokono, A. Takabayashi, S. Akimoto and A. Tanaka, "A megacomplex composed of both photosystem reaction centres in higher plants," Nat Commun, Vol. 6, Mar. 2015, pp.6675, doi: 10.1038/ncomms7675.

[9] R. Kouril, A. Zygadlo, A. A. Arteni, de C. D. Wit, J. P. Dekker, P. E. Jensen, et al., "Structural characterization of a complex of photosystem I and light-harvesting complex II of Arabidopsis thaliana," Biochemistry, Vol. 44, Aug. 2005, pp. 10935-10940.

[10] I . Jiménez, L. López, J. M. Alamillo, A. Valli and J. A. García, "Identification of a plum pox virus CI-interacting protein from chloroplast that has a negative effect in virus infection," Mol. Plant Microbe. Interact, Vol. 19, Mar. 2006, pp. 350-358.

[11] S. Mehri, K. Abnous, S. H. Mousavi and V. M. shariaty, "Neuroprotective effect of crocin on acrylamide-induced cytotoxicity in PC12 cells," Cell Mol. Neurobiol, Vol. 32, Mar. 2012, pp. 227-235, doi: 10.1007/s10571-011-9752-8.

[12] H. A. Kazi and Z. Qian, "Crocetin reduces TNBS-induced experimental colitis in mice by downregulation of NFkB," Saudi J. Gastroenterol, Vol. 15, Sep. 2009, pp. 181-187, doi: 10.4103/13193767.54750 .

[13] I. Bekesiova, J. P. Nap and L. Mlynarova, "Isolation of high quality DNA and RNA from leaves of the carnivorous plant Drosera rotundifolia," Plant Mol. Biol. Rep, Vol. 17, 1999, pp. 269-277.

[14] P. E. Jensen, L. Rosgaard, J. Knoetzel and H. V. Scheller, "Photosystem I activity is increased in the absence of the PSI-G subunit,” J. Biol. Chem, Vol. 277, Jan. 2002, pp. 2798-2803. 